\title{
Investigations on the Theory of Riemann Zeta Function III: A Simple Proof for a Restricted Lindelöf Hypothesis
}

\section{Prof. Dr. Raja Rama Gandhi ${ }^{1}$,Edigles Guedes ${ }^{2}$ and Srinivas Kishan Anapu ${ }^{3}$ \\ ${ }^{1}$ Resource person in Math for Oxford University Press, Professor in Math, BITS-Vizag. \\ ${ }^{2}$ Number Theorist, Pernambuco, Brazil. \\ ${ }^{3}$ CEO, Voice of Bigdata Solutions Pvt. Ltd., Austin, TX.}

ABSTRACT. We create new formulas for proving Lindelöf Hypothesis from Zeta Function.

\section{INTRODUCTION}

In [1], we encounter that Lindelöf, in his paper [2], showed that the function $\mu\left(\frac{1}{2}\right)$ is decreasing and convex. This led him to conjecture that $\mu\left(\frac{1}{2}\right)=0$, and consequently that

$$
\zeta\left(\frac{1}{2}+i t\right) \leq t^{\epsilon}
$$

whatever $\epsilon>0$.

In this paper, we will demonstrate that

$$
\zeta\left(\frac{1}{2}+i t\right) \leq t^{\epsilon}
$$

whatsoever $\epsilon>0$ and any $t \in \mathbb{R}_{\geq 0.30611645227149686 \ldots}$.

\section{PRELIMINARES}

In [3] we have a convergent series representation for $\zeta(s, q)$, defined when $q>-1$ and any complex $s \neq 1$, which was given by Helmut Hasse, in 1930 [4]:

$$
\zeta(s, q)=\frac{1}{s-1} \sum_{n=0}^{\infty} \frac{1}{n+1} \sum_{k=0}^{n}(-1)^{k}\left(\begin{array}{l}
n \\
k
\end{array}\right)(q+k)^{1-s} .
$$

This series converges uniformly on compact subsets of the s-plane to an entire function. The inner sum may be understood to be the $\mathrm{n} t h$ forward difference of $q^{1-s}$; i.e.,

$$
\Delta^{n} q^{1-s}=\sum_{k=0}^{n}(-1)^{n-k}\left(\begin{array}{l}
n \\
k
\end{array}\right)(q+k)^{1-s},
$$

where $\Delta$ denotes the forward difference operator. As soon, we may write

$$
\begin{aligned}
\zeta(s, q) & =\frac{1}{s-1} \sum_{n=0}^{\infty} \frac{(-1)^{n}}{n+1} \Delta^{n} q^{1-s} \\
& =\frac{1}{s-1} \frac{\log (1+\Delta)}{\Delta} q^{1-s} .
\end{aligned}
$$

In [5], we see that the complex exponentiation satisfies

$$
(a+b i)^{c+d i}=\left(a^{2}+b^{2}\right)^{(c+i d) / 2} e^{i(c+i d) \arg (a+i b)},
$$

where $\arg (z)$ denotes the complex argument. We explicitly written in terms of real and imaginary parts, as follows

$$
\begin{gathered}
(a+b i)^{c+d i}=\left(a^{2}+b^{2}\right)^{c / 2} \\
\times\left\{\cos \left[c \cdot \arg (a+i b)+\frac{1}{2} d \log \left(a^{2}+b^{2}\right)\right]+i \sin \left[c \cdot \arg (a+i b)+\frac{1}{2} d \log \left(a^{2}+b^{2}\right)\right]\right\} .
\end{gathered}
$$


THEOREM 1. Let $\operatorname{Re}(s)>0$ and $s \neq 1$, then

$$
\zeta(\mathrm{s})=\frac{2^{\mathrm{s}}}{2^{\mathrm{s}-1}}+\frac{\zeta\left(\mathrm{s}, \frac{3}{2}\right)}{2^{\mathrm{s}-1}},
$$

where $\zeta(s)$ is the Riemann zeta function and $\zeta(s, a)$ is the Hurwitz zeta function.

Proof. See [6].

\section{LEMMAS AND THEOREMS}

LEMMA 1. For $t \in \mathbb{R}_{\geq 0}$, then

$$
\begin{gathered}
\zeta\left(\frac{1}{2}+i t, \frac{3}{2}\right)=-\frac{2 t}{4 t^{2}+1} \times \sum_{n=0}^{\infty} \frac{1}{n+1} \sum_{k=0}^{n}(-1)^{k}\left(\begin{array}{l}
n \\
k
\end{array}\right)\left(\frac{2 k+3}{2}\right)^{1 / 2} \cos \left[t \log \left(\frac{2 k+3}{2}\right)\right] \\
-\frac{4 t}{4 t^{2}+1} \times \sum_{n=0}^{\infty} \frac{1}{n+1} \sum_{k=0}^{n}(-1)^{k}\left(\begin{array}{l}
n \\
k
\end{array}\right)\left(\frac{2 k+3}{2}\right)^{1 / 2} \sin \left[t \log \left(\frac{2 k+3}{2}\right)\right] \\
+\frac{2 i}{4 t^{2}+1} \times \sum_{n=0}^{\infty} \frac{1}{n+1} \sum_{k=0}^{n}(-1)^{k}\left(\begin{array}{l}
n \\
k
\end{array}\right)\left(\frac{2 k+3}{2}\right)^{1 / 2} \sin \left[t \log \left(\frac{2 k+3}{2}\right)\right] \\
-\frac{4 i t}{4 t^{2}+1} \times \sum_{n=0}^{\infty} \frac{1}{n+1} \sum_{k=0}^{n}(-1)^{k}\left(\begin{array}{l}
n \\
k
\end{array}\right)\left(\frac{2 k+3}{2}\right)^{1 / 2} \cos \left[t \log \left(\frac{2 k+3}{2}\right)\right],
\end{gathered}
$$

where $\zeta(s, a)$ is the Hurwitz zeta function.

Proof. Let $s=\frac{1}{2}+i t$ and $q=\frac{3}{2}$ in (2.1)

$$
\begin{gathered}
\zeta\left(\frac{1}{2}+i t, \frac{3}{2}\right)=\frac{2}{-1+2 i t} \sum_{n=0}^{\infty} \frac{1}{n+1} \sum_{k=0}^{n}(-1)^{k}\left(\begin{array}{l}
n \\
k
\end{array}\right)\left(\frac{2 k+3}{2}\right)^{\frac{1}{2}-i t} \\
=\frac{2}{-1+2 i t} \times\left(\frac{-1-2 i t}{-1-2 i t}\right) \sum_{n=0}^{\infty} \frac{1}{n+1} \sum_{k=0}^{n}(-1)^{k}\left(\begin{array}{l}
n \\
k
\end{array}\right)\left(\frac{2 k+3}{2}\right)^{\frac{1}{2}-i t} \\
=\frac{-2-4 i t}{4 t^{2}+1} \times \sum_{n=0}^{\infty} \frac{1}{n+1} \sum_{k=0}^{n}(-1)^{k}\left(\begin{array}{l}
n \\
k
\end{array}\right)\left(\frac{2 k+3}{2}\right)^{\frac{1}{2}-i t} .
\end{gathered}
$$

On the other hand, we evaluate, using (2.5), that

$$
\begin{gathered}
\left(\frac{2 k+3}{2}\right)^{\frac{1}{2}-i t}=\left(\frac{2 k+3}{2}\right)^{1 / 2} \times \\
\times\left\{\cos \left[\frac{1}{2} \cdot \arg \left(\frac{2 k+3}{2}\right)-t \log \left(\frac{2 k+3}{2}\right)\right]+i \sin \left[\frac{1}{2} \cdot \arg \left(\frac{2 k+3}{2}\right)-t \log \left(\frac{2 k+3}{2}\right)\right]\right\} .
\end{gathered}
$$

Since $k=0,1,2,3, \ldots$, then $\arg \left(\frac{2 k+3}{2}\right)=0$; we set this in (3.3)

$$
\begin{array}{r}
\left(\frac{2 k+3}{2}\right)^{\frac{1}{2}-i t}=\left(\frac{2 k+3}{2}\right)^{1 / 2} \times\left\{\cos \left[-t \log \left(\frac{2 k+3}{2}\right)\right]+i \sin \left[-t \log \left(\frac{2 k+3}{2}\right)\right]\right\} \\
=\left(\frac{2 k+3}{2}\right)^{1 / 2} \times\left\{\cos \left[t \log \left(\frac{2 k+3}{2}\right)\right]-i \sin \left[t \log \left(\frac{2 k+3}{2}\right)\right]\right\} .
\end{array}
$$


Substituting (3.4) in (3.2), we encounter

$$
\begin{aligned}
& \zeta\left(\frac{1}{2}+i t, \frac{3}{2}\right)=\left(\frac{-2-4 i t}{4 t^{2}+1}\right) \times \sum_{n=0}^{\infty} \frac{1}{n+1} \sum_{k=0}^{n}(-1)^{k}\left(\begin{array}{l}
n \\
k
\end{array}\right)\left(\frac{2 k+3}{2}\right)^{1 / 2} \times \\
& \times\left\{\cos \left[t \log \left(\frac{2 k+3}{2}\right)\right]-i \sin \left[t \log \left(\frac{2 k+3}{2}\right)\right]\right\} \\
& =\left(\frac{-2-4 i t}{4 t^{2}+1}\right) \times \sum_{n=0}^{\infty} \frac{1}{n+1} \sum_{k=0}^{n}(-1)^{k}\left(\begin{array}{l}
n \\
k
\end{array}\right)\left(\frac{2 k+3}{2}\right)^{1 / 2} \cos \left[t \log \left(\frac{2 k+3}{2}\right)\right] \\
& +\left(\frac{-4 t+2 i}{4 t^{2}+1}\right) \times \sum_{n=0}^{\infty} \frac{1}{n+1} \sum_{k=0}^{n}(-1)^{k}\left(\begin{array}{l}
n \\
k
\end{array}\right)\left(\frac{2 k+3}{2}\right)^{1 / 2} \sin \left[t \log \left(\frac{2 k+3}{2}\right)\right] \\
& =-\frac{2 t}{4 t^{2}+1} \times \sum_{n=0}^{\infty} \frac{1}{n+1} \sum_{k=0}^{n}(-1)^{k}\left(\begin{array}{l}
n \\
k
\end{array}\right)\left(\frac{2 k+3}{2}\right)^{1 / 2} \cos \left[t \log \left(\frac{2 k+3}{2}\right)\right] \\
& -\frac{4 i t}{4 t^{2}+1} \times \sum_{n=0}^{\infty} \frac{1}{n+1} \sum_{k=0}^{n}(-1)^{k}\left(\begin{array}{l}
n \\
k
\end{array}\right)\left(\frac{2 k+3}{2}\right)^{1 / 2} \cos \left[t \log \left(\frac{2 k+3}{2}\right)\right] \\
& -\frac{4 t}{4 t^{2}+1} \times \sum_{n=0}^{\infty} \frac{1}{n+1} \sum_{k=0}^{n}(-1)^{k}\left(\begin{array}{l}
n \\
k
\end{array}\right)\left(\frac{2 k+3}{2}\right)^{1 / 2} \sin \left[t \log \left(\frac{2 k+3}{2}\right)\right] \\
& +\frac{2 i}{4 t^{2}+1} \times \sum_{n=0}^{\infty} \frac{1}{n+1} \sum_{k=0}^{n}(-1)^{k}\left(\begin{array}{l}
n \\
k
\end{array}\right)\left(\frac{2 k+3}{2}\right)^{1 / 2} \sin \left[t \log \left(\frac{2 k+3}{2}\right)\right] \\
& =-\frac{2 t}{4 t^{2}+1} \times \sum_{n=0}^{\infty} \frac{1}{n+1} \sum_{k=0}^{n}(-1)^{k}\left(\begin{array}{l}
n \\
k
\end{array}\right)\left(\frac{2 k+3}{2}\right)^{1 / 2} \cos \left[t \log \left(\frac{2 k+3}{2}\right)\right] \\
& -\frac{4 t}{4 t^{2}+1} \times \sum_{n=0}^{\infty} \frac{1}{n+1} \sum_{k=0}^{n}(-1)^{k}\left(\begin{array}{l}
n \\
k
\end{array}\right)\left(\frac{2 k+3}{2}\right)^{1 / 2} \sin \left[t \log \left(\frac{2 k+3}{2}\right)\right] \\
& +\frac{2 i}{4 t^{2}+1} \times \sum_{n=0}^{\infty} \frac{1}{n+1} \sum_{k=0}^{n}(-1)^{k}\left(\begin{array}{l}
n \\
k
\end{array}\right)\left(\frac{2 k+3}{2}\right)^{1 / 2} \sin \left[t \log \left(\frac{2 k+3}{2}\right)\right] \\
& -\frac{4 i t}{4 t^{2}+1} \times \sum_{n=0}^{\infty} \frac{1}{n+1} \sum_{k=0}^{n}(-1)^{k}\left(\begin{array}{l}
n \\
k
\end{array}\right)\left(\frac{2 k+3}{2}\right)^{1 / 2} \cos \left[t \log \left(\frac{2 k+3}{2}\right)\right] \text {. }
\end{aligned}
$$

THEOREM 1. For $\epsilon>0$ and any $t \in \mathbb{R}_{\geq 0}$, then

$$
\zeta\left(\frac{1}{2}+i t\right) \leq t^{\epsilon}
$$

Proof. Hereinafter, we will use the reductio ad absurdum to prove (3.6).

Step 1 . We assume, by hypothesis, that

$$
\zeta\left(\frac{1}{2}+i t\right)>t^{\epsilon}
$$

whatsoever $\epsilon>0$ and any $t \in \mathbb{R}_{\geq 0}$. Let $s=\frac{1}{2}+i t$ in (2.6) 


$$
\zeta\left(\frac{1}{2}+i t\right)=\frac{2^{\frac{1}{2}+i t}}{2^{\frac{1}{2}+i t}-1}+\frac{\zeta\left(\frac{1}{2}+i t, \frac{3}{2}\right)}{2^{\frac{1}{2}+i t}-1} .
$$

Substituting the right-hand side of (3.8) in (3.7), we obtain

$$
\frac{2^{\frac{1}{2}+i t}}{2^{\frac{1}{2}+i t}-1}+\frac{\zeta\left(\frac{1}{2}+i t, \frac{3}{2}\right)}{2^{\frac{1}{2}+i t}-1}>t^{\epsilon} \Rightarrow 2^{i t}+\frac{t^{\epsilon}}{\sqrt{2}}+\frac{\zeta\left(\frac{1}{2}+i t, \frac{3}{2}\right)}{\sqrt{2}}>2^{i t} t^{\epsilon} .
$$

Step 2. We defined

$$
C(t):=\sum_{n=0}^{\infty} \frac{1}{n+1} \sum_{k=0}^{n}(-1)^{k}\left(\begin{array}{l}
n \\
k
\end{array}\right)\left(\frac{2 k+3}{2}\right)^{1 / 2} \cos \left[t \log \left(\frac{2 k+3}{2}\right)\right]
$$

and

$$
S(t):=\sum_{n=0}^{\infty} \frac{1}{n+1} \sum_{k=0}^{n}(-1)^{k}\left(\begin{array}{l}
n \\
k
\end{array}\right)\left(\frac{2 k+3}{2}\right)^{1 / 2} \sin \left[t \log \left(\frac{2 k+3}{2}\right)\right]
$$

using this in (3.1)

$$
\begin{aligned}
\zeta\left(\frac{1}{2}+i t, \frac{3}{2}\right) & =-\frac{2 t}{4 t^{2}+1} C(t)-\frac{4 t}{4 t^{2}+1} S(t)+\frac{2 i}{4 t^{2}+1} S(t)-\frac{4 i t}{4 t^{2}+1} C(t) \\
= & -\frac{2 t}{4 t^{2}+1} \cdot[C(t)+2 S(t)]+\frac{2 i}{4 t^{2}+1}[S(t)-2 t C(t)] .
\end{aligned}
$$

Step 3. We use (2.5) for evaluate $2^{i t}$, as follows

$$
2^{i t}=\cos (t \log 2)+i \sin (t \log 2) .
$$

Step 4. From (3.9), (3.12) and (3.13), we obtain

$$
\begin{aligned}
& \cos (t \log 2)+i \sin (t \log 2)+\frac{t^{\epsilon}}{\sqrt{2}}-\frac{\sqrt{2} t}{4 t^{2}+1} \cdot[C(t)+2 S(t)]+\frac{\sqrt{2} i}{4 t^{2}+1}[S(t)-2 t C(t)]> \\
& \cos (t \log 2) \cdot t^{\epsilon}+i \sin (t \log 2) \cdot t^{\epsilon}
\end{aligned}
$$

so

$$
\begin{aligned}
& \cos (t \log 2)+\frac{t^{\epsilon}}{\sqrt{2}}-\frac{\sqrt{2} t}{4 t^{2}+1} \cdot[C(t)+2 S(t)]+i \sin (t \log 2)+\frac{\sqrt{2} i}{4 t^{2}+1}[S(t)-2 t C(t)]> \\
& \cos (t \log 2) \cdot t^{\epsilon}+i \sin (t \log 2) \cdot t^{\epsilon}
\end{aligned}
$$

Step 5. We compare the real and imaginary part separately of (3.15). Therefore, for the real part, we find

$$
\begin{gathered}
\cos (t \log 2)+\frac{t^{\epsilon}}{\sqrt{2}}-\frac{\sqrt{2} t}{4 t^{2}+1} \cdot[C(t)+2 S(t)]>\cos (t \log 2) \cdot t^{\epsilon} \\
\Rightarrow \cos (t \log 2)+\frac{t^{\epsilon}}{\sqrt{2}}>\cos (t \log 2) \cdot t^{\epsilon}+\frac{\sqrt{2} t}{4 t^{2}+1} \cdot[C(t)+2 S(t)] \\
\Rightarrow \frac{\cos (t \log 2)}{t^{\epsilon}}+\frac{1}{\sqrt{2}}>\cos (t \log 2)+\frac{\sqrt{2} t}{\left(4 t^{2}+1\right) t^{\epsilon}} \cdot[C(t)+2 S(t)] .
\end{gathered}
$$

and, for the imaginary part, we encounter

$$
\begin{aligned}
& \sin (t \log 2)+\frac{\sqrt{2}}{4 t^{2}+1}[S(t)-2 t C(t)]>\sin (t \log 2) \cdot t^{\epsilon} \\
& \Rightarrow \sin (t \log 2)+\frac{\sqrt{2}}{4 t^{2}+1} S(t)>\sin (t \log 2) \cdot t^{\epsilon}+\frac{2 t \sqrt{2}}{4 t^{2}+1} C(t)
\end{aligned}
$$


Step 6. Real part. We divide the inequality (3.16) by $t^{2+\epsilon}$

$$
\frac{\cos (t \log 2)}{t^{2(\epsilon+1)}}+\frac{1}{t^{2+\epsilon} \sqrt{2}}>\frac{\cos (t \log 2)}{t^{2+\epsilon}}+\frac{\sqrt{2} t}{\left(4 t^{2}+1\right) t^{2(\epsilon+1)}} \cdot[C(t)+2 S(t)] .
$$

We evaluate the limit when $t \rightarrow+\infty$ of (3.18)

$$
\lim _{t \rightarrow+\infty}\left[\frac{\cos (t \log 2)}{t^{2(\epsilon+1)}}+\frac{1}{t^{2+\epsilon} \sqrt{2}}\right]>\lim _{t \rightarrow+\infty}\left\{\frac{\cos (t \log 2)}{t^{2+\epsilon}}+\frac{\sqrt{2} t}{\left(4 t^{2}+1\right) t^{2(\epsilon+1)}} \cdot[C(t)+2 S(t)]\right\}
$$

$\lim _{t \rightarrow+\infty}\left[\frac{\cos (t \log 2)}{t^{2(\epsilon+1)}}\right]+\lim _{t \rightarrow+\infty}\left(\frac{1}{t^{2+\epsilon} \sqrt{2}}\right)>\lim _{t \rightarrow+\infty}\left[\frac{\cos (t \log 2)}{t^{2+\epsilon}}\right]+\lim _{t \rightarrow+\infty}\left\{\frac{\sqrt{2} t}{\left(4 t^{2}+1\right) t^{2(\epsilon+1)}}\right.$. $[C(t)+2 S(t)]\}$.

Note 1: We calculate, for any $\epsilon>0$ and $\left.k=0,1,2,3, \ldots: 1^{\circ}\right)$ when $t \rightarrow+\infty$, then $\left.\frac{\cos (t \log 2)}{t^{2(\epsilon+1)}} \rightarrow 0 ; 2 .^{\circ}\right)$ when $t \rightarrow+\infty$, then $\left.\frac{1}{t^{2+\epsilon} \sqrt{2}} \rightarrow 0 ; 3 .^{\circ}\right)$ when $t \rightarrow+\infty$, then $\left.\frac{\cos (t \log 2)}{t^{2+\epsilon}} \rightarrow 0 ; 4 .^{\circ}\right)$ and when $t \rightarrow+\infty$, then $\frac{\sqrt{2} t}{\left(4 t^{2}+1\right) t^{2(\epsilon+1)}} \cdot\left\{\cos \left[t \log \left(\frac{2 k+3}{2}\right)\right]+2 \sin \left[t \log \left(\frac{2 k+3}{2}\right)\right]\right\} \rightarrow 0$. So, our hypothesis is false, because $0 \ngtr 0$; but, $0=0$. We conclude that our hypothesis for the real part is false.

Step 7. Imaginary part. We divide the inequality (3.17) by $t^{2+\epsilon}$

$$
\begin{gathered}
\frac{\sin (t \log 2)}{t^{2+\epsilon}}+\frac{\sqrt{2}}{\left(4 t^{2}+1\right) t^{2+\epsilon}} S(t)>\frac{\sin (t \log 2) \cdot t^{\epsilon}}{t^{2+\epsilon}}+\frac{2 t \sqrt{2}}{\left(4 t^{2}+1\right) t^{2+\epsilon}} C(t) \\
\Rightarrow \frac{\sin (t \log 2)}{t^{2+\epsilon}}+\frac{\sqrt{2}}{\left(4 t^{2}+1\right) t^{2+\epsilon}} S(t)>\frac{\sin (t \log 2)}{t^{2}}+\frac{2 t \sqrt{2}}{\left(4 t^{2}+1\right) t^{2+\epsilon}} C(t) .
\end{gathered}
$$

We evaluate the limit when $t \rightarrow+\infty$ of (3.20)

$$
\lim _{t \rightarrow+\infty}\left[\frac{\sin (t \log 2)}{t^{2+\epsilon}}+\frac{\sqrt{2}}{\left(4 t^{2}+1\right) t^{2+\epsilon}} S(t)\right]>\lim _{t \rightarrow+\infty}\left[\frac{\sin (t \log 2)}{t^{2}}+\frac{2 t \sqrt{2}}{\left(4 t^{2}+1\right) t^{2+\epsilon}} C(t)\right]
$$

$\lim _{t \rightarrow+\infty}\left[\frac{\sin (t \log 2)}{t^{2+\epsilon}}\right]+\lim _{t \rightarrow+\infty}\left[\frac{\sqrt{2}}{\left(4 t^{2}+1\right) t^{2+\epsilon}} S(t)\right]>\lim _{t \rightarrow+\infty}\left[\frac{\sin (t \log 2)}{t^{2}}\right]+$ $\lim _{t \rightarrow+\infty}\left[\frac{2 t \sqrt{2}}{\left(4 t^{2}+1\right) t^{2+\epsilon}} C(t)\right]$.

Note 2: We calculate, for any $\epsilon>0$ and $\left.k=0,1,2,3, \ldots: 1^{\circ}{ }^{\circ}\right)$ when $t \rightarrow+\infty$, then $\left.\frac{\sin (t \log 2)}{t^{2+\epsilon}} \rightarrow 0 ; 2 .^{\circ}\right)$ when $t \rightarrow+\infty$, then $\left.\frac{\sqrt{2}}{\left(4 t^{2}+1\right) t^{2+\epsilon}} \sin \left[t \log \left(\frac{2 k+3}{2}\right)\right] \rightarrow 0 ; 3 .^{\circ}\right)$ when $t \rightarrow+\infty$, then $\left.\frac{\sin (t \log 2)}{t^{2}} \rightarrow 0 ; 4 .^{\circ}\right)$ and when $t \rightarrow+\infty$, then $\frac{2 t \sqrt{2}}{\left(4 t^{2}+1\right) t^{2+\epsilon}} \cos \left[t \log \left(\frac{2 k+3}{2}\right)\right] \rightarrow 0$. So, our hypothesis is false, because $0 \ngtr 0$; but, $0=0$. We conclude that our hypothesis for the imaginary part is false.

Step 8. We evaluate the function $\max _{t \in \mathbb{R}_{\geq 0}, k \in \mathbb{Z}_{\geq 0}}\left\{\cos \left[t \log \left(\frac{2 k+3}{2}\right)\right]\right\}$. For $k=0$, then $\max _{t \in \mathbb{R} \geq 0}\left\{\cos \left[t \log \left(\frac{3}{2}\right)\right]\right\}=1$, when $t=0$; for $k=1$, then $\max _{t \in \mathbb{R}_{\geq 0}}\left\{\cos \left[t \log \left(\frac{5}{2}\right)\right]\right\}=1$, when $t=0$; for $k=2$, then $\max _{t \in \mathbb{R}_{\geq 0}}\left\{\cos \left[t \log \left(\frac{7}{2}\right)\right]\right\}=1$, when $t=0$; and so on. We easily deduzimos que $\max _{t \in \mathbb{R}_{\geq 0}, k \in \mathbb{Z}_{\geq 0}}\left\{\cos \left[t \log \left(\frac{2 k+3}{2}\right)\right]\right\}=1$, when $t=0$. So, 
$\sum_{n=0}^{\infty} \frac{1}{n+1} \sum_{k=0}^{n}(-1)^{k}\left(\begin{array}{l}n \\ k\end{array}\right)\left(\frac{2 k+3}{2}\right)^{1 / 2} \cos \left[t \log \left(\frac{2 k+3}{2}\right)\right]=: C(t)<$

$\sum_{n=0}^{\infty} \frac{1}{n+1} \sum_{k=0}^{n}(-1)^{k}\left(\begin{array}{l}n \\ k\end{array}\right)\left(\frac{2 k+3}{2}\right)^{1 / 2} \max _{t \in \mathbb{R}_{\geq 0}, k \in \mathbb{Z}_{\geq 0}}\left\{\cos \left[t \log \left(\frac{2 k+3}{2}\right)\right]\right\}=$

$\sum_{n=0}^{\infty} \frac{1}{n+1} \sum_{k=0}^{n}(-1)^{k}\left(\begin{array}{l}n \\ k\end{array}\right)\left(\frac{2 k+3}{2}\right)^{1 / 2}$,

where $\sum_{n=0}^{\infty} \frac{1}{n+1} \sum_{k=0}^{n}(-1)^{k}\left(\begin{array}{l}n \\ k\end{array}\right)\left(\frac{2 k+3}{2}\right)^{1 / 2}$ tends to $1.0095 \ldots$ very slowly. Simplifying, we find

$$
1.0095 \ldots>C(t)
$$

On the other hand, continuando with the evaluate for the function $\min _{t \in \mathbb{R}_{\geq 0}, k \in \mathbb{Z}_{\geq 0}}\left\{\cos \left[t \log \left(\frac{2 k+3}{2}\right)\right]\right\}$. For $k=0$, then $\min _{t \in \mathbb{R}_{\geq 0}}\left\{\cos \left[t \log \left(\frac{3}{2}\right)\right]\right\}=\cos (\pi)=-1$, when $t=\frac{\pi}{\log 3-\log 2}=7.74812083893 \ldots$; for $k=1$, then $\min _{t \in \mathbb{R}_{\geq 0}}\left\{\cos \left[t \log \left(\frac{5}{2}\right)\right]\right\}=\cos (\pi)=$ -1 , when $t=\frac{\pi}{\log 5-\log 2}=3.42859809044 \ldots ;$ for $k=2$, then $\min _{t \in \mathbb{R}_{\geq 0}}\left\{\cos \left[t \log \left(\frac{7}{2}\right)\right]\right\}=$ $\cos (\pi)=-1$, when $t=\frac{\pi}{\log 7-\log 2}=2.50773109726 \ldots$; and so on. We easily deduzimos que $\min _{t \in \mathbb{R}_{\geq 0}, k \in \mathbb{Z}_{\geq 0}}\left\{\cos \left[t \log \left(\frac{2 k+3}{2}\right)\right]\right\}=\cos (\pi)=-1$, when $t=\frac{\pi}{\log (2 k+3)-\log 2}$, for $k=0,1,2,3, \ldots$ So,

$\sum_{n=0}^{\infty} \frac{1}{n+1} \sum_{k=0}^{n}(-1)^{k}\left(\begin{array}{l}n \\ k\end{array}\right)\left(\frac{2 k+3}{2}\right)^{1 / 2} \cos \left[t \log \left(\frac{2 k+3}{2}\right)\right]=: C(t)>>$

$\sum_{n=0}^{\infty} \frac{1}{n+1} \sum_{k=0}^{n}(-1)^{k}\left(\begin{array}{l}n \\ k\end{array}\right)\left(\frac{2 k+3}{2}\right)^{1 / 2} \min _{t \in \mathbb{R}_{\geq 0}, k \in \mathbb{Z}_{\geq 0}}\left\{\cos \left[t \log \left(\frac{2 k+3}{2}\right)\right]\right\}=$

$-\sum_{n=0}^{\infty} \frac{1}{n+1} \sum_{k=0}^{n}(-1)^{k}\left(\begin{array}{l}n \\ k\end{array}\right)\left(\frac{2 k+3}{2}\right)^{1 / 2}$,

where $\sum_{n=0}^{\infty} \frac{1}{n+1} \sum_{k=0}^{n}(-1)^{k}\left(\begin{array}{l}n \\ k\end{array}\right)\left(\frac{2 k+3}{2}\right)^{1 / 2}$ tends to $1.0095 \ldots$ very slowly. Simplifying, we have

$$
C(t)>-1.0095
$$

From (3.23) and (3.25), it follows that

$$
-1.0095 \ldots<C(t)<1.0095 \ldots
$$

Step 10. From (3.16) and (3.17), we have the following system of inequalities

$$
\left\{\begin{array}{c}
\cos (t \log 2)+\frac{t^{\epsilon}}{\sqrt{2}}>\cos (t \log 2) \cdot t^{\epsilon}+\frac{\sqrt{2} t}{4 t^{2}+1} \cdot[C(t)+2 S(t)] \\
\sin (t \log 2)+\frac{\sqrt{2} S(t)}{4 t^{2}+1}>\sin (t \log 2) \cdot t^{\epsilon}+2 t \frac{\sqrt{2} C(t)}{4 t^{2}+1}
\end{array}\right.
$$

Dividing both the members of (3.27) by $t^{\epsilon}$, we encounter

$$
\left\{\begin{array}{c}
\frac{\cos (t \log 2)}{t^{\epsilon}}+\frac{1}{\sqrt{2}}>\cos (t \log 2)+\frac{\sqrt{2} t}{t^{\epsilon}\left(4 t^{2}+1\right)} \cdot[C(t)+2 S(t)] \\
\frac{\sin (t \log 2)}{t^{\epsilon}}+\frac{\sqrt{2} S(t)}{t^{\epsilon}\left(4 t^{2}+1\right)}>\sin (t \log 2)+\frac{2 \sqrt{2} t C(t)}{t^{\epsilon}\left(4 t^{2}+1\right)}
\end{array}\right.
$$

From second inequality of (3.28), we find

$$
\frac{\sqrt{2} S(t)}{t^{\epsilon}\left(4 t^{2}+1\right)}>\sin (t \log 2)-\frac{\sin (t \log 2)}{t^{\epsilon}}+\frac{2 \sqrt{2} t C(t)}{t^{\epsilon}\left(4 t^{2}+1\right)}
$$


From first inequality of (3.28) and the right-hand side of (3.29), we have

$$
\begin{gathered}
\frac{\cos (t \log 2)}{t^{\epsilon}}+\frac{1}{\sqrt{2}}>\cos (t \log 2)+\frac{\sqrt{2} t}{t^{\epsilon}\left(4 t^{2}+1\right)} \cdot[C(t)+2 S(t)] \\
=\cos (t \log 2)+t \cdot\left[\frac{\sqrt{2} C(t)}{t^{\epsilon}\left(4 t^{2}+1\right)}+2 \frac{\sqrt{2} S(t)}{t^{\epsilon}\left(4 t^{2}+1\right)}\right] \\
>\cos (t \log 2)+t \cdot\left[\frac{\sqrt{2} C(t)}{t^{\epsilon}\left(4 t^{2}+1\right)}+2 \sin (t \log 2)-2 \frac{\sin (t \log 2)}{t^{\epsilon}}+\frac{4 \sqrt{2} t C(t)}{t^{\epsilon}\left(4 t^{2}+1\right)}\right] \\
=\cos (t \log 2)+2 t \sin (t \log 2)-2 t \frac{\sin (t \log 2)}{t^{\epsilon}}+\frac{\sqrt{2} t(4 t+1) C(t)}{t^{\epsilon}\left(4 t^{2}+1\right)}
\end{gathered}
$$

Consequently,

$\frac{\cos (t \log 2)}{t^{\epsilon}}+\frac{1}{\sqrt{2}}>\cos (t \log 2)+2 t \sin (t \log 2)-2 t \frac{\sin (t \log 2)}{t^{\epsilon}}+\frac{\sqrt{2} t(4 t+1) C(t)}{t^{\epsilon}\left(4 t^{2}+1\right)}$.

Thus,

$$
\frac{t^{\epsilon}\left(4 t^{2}+1\right)}{\sqrt{2} t(4 t+1)}\left[\frac{\cos (t \log 2)}{t^{\epsilon}}-\cos (t \log 2)-2 t \sin (t \log 2)+2 t \frac{\sin (t \log 2)}{t^{\epsilon}}+\frac{1}{\sqrt{2}}\right]>C(t) .
$$

Step 10. We divide (3.32) by (3.23)

$$
\begin{gathered}
\frac{\cos (t \log 2)}{t^{\epsilon}}-\cos (t \log 2)-2 t \sin (t \log 2)+2 t \frac{\sin (t \log 2)}{t^{\epsilon}}+\frac{1}{\sqrt{2}}>1.0095 \ldots \frac{\sqrt{2} t(4 t+1)}{t^{\epsilon}\left(4 t^{2}+1\right)} \\
\frac{\cos (t \log 2)}{t^{\epsilon}}+2 t \frac{\sin (t \log 2)}{t^{\epsilon}}+\frac{1}{\sqrt{2}}>\cos (t \log 2)+2 t \sin (t \log 2)+1.0095 \ldots \frac{\sqrt{2} t(4 t+1)}{t^{\epsilon}\left(4 t^{2}+1\right)}
\end{gathered}
$$

Multiplying (3.33) by $t^{\epsilon}$, we find

$$
\cos (t \log 2)+2 t \sin (t \log 2)>\left[\cos (t \log 2)+2 t \sin (t \log 2)+1.0095 \ldots \frac{\sqrt{2} t(4 t+1)}{4 t^{2}+1}-\frac{1}{\sqrt{2}}\right] t^{\epsilon} .
$$

From (3.34), we deduce that

$\cos (t \log 2)+2 t \sin (t \log 2)>\left[\cos (t \log 2)+2 t \sin (t \log 2)+1.0095 \ldots \frac{\sqrt{2} t(4 t+1)}{4 t^{2}+1}-\frac{1}{\sqrt{2}}\right] t^{\epsilon}>$ $\left[\cos (t \log 2)+2 t \sin (t \log 2)+1.0095 \ldots \frac{\sqrt{2} t(4 t+1)}{4 t^{2}+1}-\frac{1}{\sqrt{2}}\right] t^{0}=\cos (t \log 2)+2 t \sin (t \log 2)+$ $1.0095 \ldots \frac{\sqrt{2} t(4 t+1)}{4 t^{2}+1}-\frac{1}{\sqrt{2}}$.

It is easy to see that: for $1.0095 \ldots \frac{\sqrt{2} t(4 t+1)}{4 t^{2}+1} \geq \frac{1}{\sqrt{2}}$, the inequality (3.35) is false.

Note 3: For any $t \in \mathbb{R}^{+}$and $t \geq 0.30611645227149686 \ldots$, then the inequality (3.40) is false. So, our hypothesis is false.

Step 11. Thus, from Notes 1,2 and 3, we show that $\zeta\left(\frac{1}{2}+i t\right) \leq t^{\epsilon}$, for whatsoever $\epsilon>0$ and any $t \in \mathbb{R}_{\geq 0.30611645227149686 \ldots}$.

\section{ACKNOWLEDGMENTS}

The first author, Edigles Guedes, thank Prof. Dr. K. Raja Rama Gandhi and your society for their encouragement and support during the development of this paper. 


\section{REFERENCES}

[1] http://fr.wikipedia.org/wiki/Histoire_de_la_fonction_zêta_de_Riemann, available in May 19, 2013.

[2] E. Lindelöf, Quelques remarques sur la croissance de la function $\zeta(s)$, Bulletin des sciences mathématiques, 2. a série, vol. 3, décembre 1908, p. 341-356.

[3] http://en.wikipedia.org/wiki/Hurwitz_zeta_function\#cite_note-1, available in May 22, 2013.

[4] Hasse, Helmut (1930), Ein Summierungsverfahren für die Riemannsche $\zeta$-Reihe, Mathematische Zeitschrift, 32 (1): 458-464.

[5] Weisstein, Eric W., “Complex Exponentiation”. From MathWorld - A Wolfram Web Resource. http://mathworld.wolfram.com/ComplexExponentiation.html.

[6] Edigles Guedes and Prof. Dr. Raja Rama Gandhi, Investigations on the Theory of Riemann Zeta Function I: New Functional Equation, Integral Representation and Laurent Expansion for Riemann Zeta Function, May 1, 2013. 